\title{
Reseña bibliográfica de: Stray, C. (2018). Classics in Britain: Scholarship, Education, and Publishing 1800-2000. Oxford: Oxford University Press. xxvi, 385 pp.
}

Palabras claves: Erudición - Educación - Publicación - Estudios Clásicos

Keywords: Scholarship - Education - Publishing - Classics

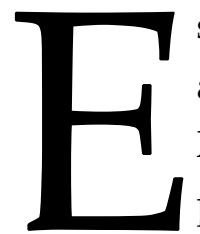

ste libro reúne una serie de artículos de Christopher Stray sobre varios aspectos de la enseñanza de los estudios clásicos y la erudición en los siglos XIX y XX en Gran Bretaña. A excepción de los capítulos 3 y 8, que son publicados aquí por primera vez, los restantes artículos vieron la luz previamente en otros libros y revistas. Sin embargo, en palabras del autor, este libro busca ser más que la suma de las partes. Incluye una bibliografía completa y un útil index nominum, que facilita mucho la tarea de consultar un libro voluminoso con información importante.

Este volumen único, bien ilustrado y producido de manera atractiva, resume y refleja el trabajo de una voz destacada en la historia de los estudios clásicos en Gran Bretaña. Los temas abarcan desde la enseñanza en el aula escolar hasta la política en las universidades y desde los usos sociales del conocimiento clásico hasta la publicación de libros de texto y manuales. Aunque el volumen en su conjunto tiene un enfoque dirigido de forma especial hacia el papel de los libros y las revistas en la recepción de los clásicos, los capítulos también exploran fuentes anecdóticas y documentales para ofrecer una imagen vívida de los rincones más oscuros del mundo de los académicos, maestros y alumnos en un largo periodo de tiempo entre los siglos XIX y XX.

El libro está dividido en tres partes. La primera muestra la utilidad del análisis comparativo de las instituciones universitarias, centrándose en los casos de Oxford y Cambridge en particular. La segunda, por su parte, analiza el papel transformador de la impresión y la publicación, así como también la historia de dos importantes instituciones, la Sociedad Helénica (1879) y la Asociación Clásica (1903), en relación directa con el lugar cambiante de los clásicos en la sociedad británica. La tercera, finalmente, se centra en la pedagogía, examina los libros de texto y la actividad en el aula y hace hincapié en la naturaleza dialéctica de la recepción, como lo demuestra el autor a partir de los escritos de los alumnos en los textos de estudio, que revelan la existencia de un espacio para la resistencia a las lecciones de sus maestros.

De manera aislada, estos capítulos se muestran interesantes, pero juntos ofrecen una visión general e inigualable de la historia y la sociología de la educación clásica y la 
erudición ${ }^{1}$ entre 1800 y 2000. Christopher Stray se encarga de transitar los caminos difíciles y, a veces, abandonados a la mitad por los que el conocimiento de los estudios clásicos ha llegado a la situación actual. Puede ser relevante tener en cuenta, sin embargo, que esta no es propiamente una historia de la erudición clásica. El contenido de algunos de los capítulos se superpone inevitablemente.

La primera parte ("Scholarship and Institutions") contiene cinco capítulos, comenzando con "Purity in danger". Allí, el autor explora el interés puesto en la crítica textual (ejemplificada aquí por Richard Shilleto, conocido filólogo de Cambridge de mediados del siglo XIX) utilizada como arma contra la defensa de la figura de Cleón y la crítica a Tucídides, que George Grote había sostenido en su A History of Greece. Stray muestra de forma cabal los clivajes que existían en la Universidad de Cambridge en ese momento, sobre todo en torno al sentido y alcance del estudio del griego y la literatura clásica en el marco de las reformas educativas impulsadas y las comisiones reales de revisión de planes. Muchos filólogos de la universidad, de los cuales Shilleto puede ser visto como un modelo, se opusieron a lo que veían como una amenaza en contra de la filología "pura" del porsonianismo. ${ }^{2}$ Creían, en ese sentido, que el nuevo modelo de abordaje del mundo antiguo podía representar una dilución del griego en la historia y la arqueología. Hay mucho más en este capítulo denso que requiere y merece mucha atención, y gran parte tiene resonancia y relevancia hoy.

El capítulo 2 ("Curriculum and style in the Collegiate University") compara y contrasta el desarrollo en el siglo XIX del currículum de estudios clásicos en Oxford y Cambridge. En Oxford, los estudios clásicos eran dominantes, mientras que, en Cambridge, el Tripos clásico emergió desde las sombras de un curso bien establecido y prestigioso de matemáticas y ganó autonomía solamente hacia finales de la década de 1850. Se muestra, entonces, de qué forma las decisiones tomadas en el siglo XIX han influido y seguido en vigencia en gran medida hasta nuestros días.

En el capítulo 3 (“Thomas Gaisford: Legion, Legend, Lexicographer"), Stray nos da una descripción mucho más amplia de la que jamás se haya visto de un hombre particular (Gaisford), cuyas actividades mostraron una energía inmensa, con su trabajo en el llamado Etymologicon Magnum, en el Hephaestion, Clarendon Press, la Biblioteca Bodleiana, como decano del Christ Church, con el diccionario Liddell \& Scott o como autor muchas veces citado. Stray insiste en la importancia del contexto en el estudio de la erudición, que ha tendido, más bien, a describir grandes figuras de forma aislada y agrega que "la vida contextual de los sabios" debería tratar no solo la interacción entre ellos, sino también los cambios en las instituciones académicas y en la sociedad en general.

En el capítulo 4 ("The Rise and Fall of Porsoniasm"), Stray está preocupado por el "fenómeno de la colaboración informal y las redes académicas", y las solidaridades y

\footnotetext{
${ }^{1}$ En el libro, aparece frecuentemente la palabra "scholarship", un término propio de la cultura británica que no tiene un equivalente preciso en español. Aquí optamos por el término "erudición".

2 Una palabra acuñada por Elmsley.
} 
los conflictos que rodearon la práctica del estilo porsoniano, más que por la erudición textualmente dirigida. Stray menciona la Ley de Porson sin agregar que no se sigue invariablemente. Los principales seguidores de la perspectiva de Porson fueron Elmsley, Dobree, Monk y Blomfield; entre los oponentes, considerados inferiores, se encontraban Samuel Parr, Edmund Barker y Samuel Butler, cuyo variorum Aeschylus recibió un trato duro. La "caída", dice Stray, fue provocada por la emergencia de perspectivas más amplias sobre la erudición. En efecto, durante los años del "Porsonianismo", el Trinity College de Cambridge tuvo menos prominencia, pero también menos contacto con universidades extranjeras en estudios clásicos. La importancia de este largo capítulo, con su ejemplar provisión de detalles, nos muestra cómo el culto al porsonianismo fue el producto de una confluencia de elementos dispares y la influencia que tuvo sobre los teólogos anglicanos quienes siguieron su ejemplo académico.

El capítulo 5 ("Renegotiating Classics. The Politics of Curricular Reform in Late Victorian Cambridge") puede resumirse, en palabras del autor, en un intento por explicar "la aparición de un patrón distintivo de organización curricular que perdura hasta el día de hoy".

La segunda sección ("Scholarship and Publishing") incluye especialmente muchos detalles sobre la multiplicidad de reseñas, muchas de ellas de corta duración, o incluso sin trascendencia, que infestaron los comienzos del siglo XIX. En el capítulo 6 ("Politics, Culture, and Scholarship") se examina, entre otras cosas, el papel de los clásicos en las escuelas públicas en su relación con las diferentes perspectivas de dos de las principales revistas de reseñas críticas: el Edinburgh Review y el Quarterly Review. Stray también aborda la gran diferencia de estilo que existió entre Cambridge y Oxford y las cinco universidades escocesas, así como también incluso entre las dos universidades inglesas.

El capítulo 7 ("From one Museum to Another") se dedica principalmente a entrar en más detalles sobre las revistas, enumerando once que despegaron y una que no lo hizo, el Museum Academicum, a pesar del respaldo de Benjamin Jowett, Henry Liddell, Robert Scott y Arthur Stanley. Además, aquí notamos que solo tres de las revistas de hoy surgieron en el siglo XIX (The Journal of Hellenic Studies, The Classical Review y The Classical Quarterly) y que el The Journal of Philology se publicó entre 1868-1921,3 agregando que cuando la Universidad de Cambridge decidió no renovar una suscripción masiva, cerró. Aunque, sin duda, la duplicación de los precios minoristas entre 1914 y 1918 debió haber sido un factor significativo.

En el capítulo 8 ("The Classical Review and its Precursors"), se indaga sobre una serie de revistas especializadas en estudios clásicos que no tuvieron continuidad $\mathrm{y}$, sobre todo, sobre el papel central que tuvo Joseph Mayor en la fundación de la revista The Classical Review. Stray señala que, este papel central, se debió al clima de época. La

\footnotetext{
${ }^{3}$ o 1920: ver p. 236.
} 
relajación de las restricciones religiosas y las reglas de celibato implicaban que había "poderosos apoyos" para la creación de una comunidad disciplinaria que pudiera suministrar tanto a escritores y editores, así como a lectores.

A continuación, se presentan dos capítulos sobre individuos: el capítulo 9 sobre Sir William Smith ("Sir William Smith and his Dictionaries") y el 10 sobre Sir Richard Jebb ("Jebb's Sophocles: An Edition and its Maker"). Smith, caracterizado por su energía y asiduidad, todavía es conocido por su producción enciclopédica. Acerca de esto, Stray enumera siete diccionarios que "reflejaron la ampliación de la base social e institucional de la erudición más allá de los enclaves anglicanos de Oxbridge para los disidentes y círculos seculares de Londres y las provincias". Sobre Jebb, Stray resume que su Sófocles era un "gran proyecto victoriano tardío impulsado por la concepción idealista del editor de la Grecia clásica y Sófocles", destacándose su creencia de que tal concepto seguía siendo válido en una era moderna de transición. Stray, resalta el hecho de la perdurabilidad de este trabajo como una de sus principales virtudes.

El capítulo 11 ("Promoting and Defending") nos lleva al momento en que la enseñanza obligatoria del griego se vio amenazada (por supuesto, resistió ferozmente hasta su abolición en 1920) en Oxbridge y en Londres y trata sobre los pormenores de la defensa del helenismo como un conocimiento que venía a reemplazar la fe cristiana. En cuanto a esto, el joven Gilbert Murray dijo en su conferencia inaugural en Glasgow: "Grecia, no el griego, es el verdadero tema de nuestro estudio". Este fue el trasfondo de la fundación de la Sociedad Helénica y, veinte años después, de la Asociación Clásica en el medio académico del University College de Londres, con J. P. Postgate como el motor principal. Por lo tanto, en el momento de la aparición del Comité de Clásicos en Educación (1919), Stray nos muestra una institución llena de "cuerpos" clásicos: las dos Sociedades, las Escuelas de Atenas y Roma, la Asociación Clásica y The Classical Review y The Classical Quarterly.

El capítulo 12 ("Scholars, Gentlemen, and Schoolboys") es de interés más específico, dado que se concentra en el latín, "incrustado en el plan de estudios de las escuelas de gramática", al tiempo que estudia el problema hasta 1960, en que Oxbridge abolió el obligatorio nivel de latín como requisito para la admisión en las universidades.

La tercera parte ("Schools and Schoolbooks") consta de seis capítulos. El capítulo 13 ("Paper Wraps Stone: The beginnings of Educational Lithography") pone el foco en la razón por la cual la técnica litográfica fue utilizada a comienzos del siglo XIX: por su bajo costo y por la facilidad para producir tiradas cortas y sin contenido textual. El capítulo 14 (“John Taylor and 'Locke's Classical System'”) nos explica un sistema que es básicamente un conjunto de traducciones interlineales de autores latinos y griegos (luego se le agregó el hebreo y el alemán), junto con gramáticas latinas y griegas.

El capítulo 15 ("Schoolboys and Gentlemen: Classical Pedagogy and Authority in the English Public School") nos presenta a un colegio Winchester que, dice Stray, es "típico de una tendencia contemporánea generalizada, que está íntimamente vinculada con los discursos ingleses de autoridad y libertad del siglo XIX". La sección encabezada 
por Horae Mush (rianae) es, en cualquier caso, muy divertida, y brinda una "rara oportunidad de ver la forma en que se negocia la autoridad pedagógica". El capítulo 16 ("Edward Adolf Sonnenschein and the Politics of Linguistic Authority in England, 1880-1930") brinda un análisis acerca de la campaña llevada a cabo por Sonnenschein en defensa de descripciones funcionales generalizadas de la estructura lingüística contra los relativismos formales vernáculos adoptados por el principal oponente de Sonnenschein, el lingüista danés Otto Jespersen. Esta campaña pertenece a un periodo de transición de la sociedad inglesa a la modernidad como un Estado nación del siglo XX.

En el capítulo 17 ("Primers, Publishing, and Politics: The Classical Textbooks of Benjamin Hall Kennedy"), aprendemos por qué medios, y a pesar de mucha oposición, Kennedy Public School Latin Primer llegó a asumir la posición dominante (y rentable) que ocuparía durante tantos años. Además, nos permite conocer cómo Benjamin Hall Kennedy fue ayudado por el trabajo, no reconocido, de sus dos hijas, que fueron centrales en la producción de la cartilla latina revisada. Cabe destacar que el manual básico original fue encargado por los directores de las nueve escuelas "Clarendon", siendo el propio Kennedy uno de ellos hasta 1866.

En el capítulo final, el 18, aprendemos sobre la relevancia de la cartilla antes mencionada en The Turn of the Screw de Britten, como "un símbolo del aprendizaje escolar masculino convencional". Stray remarca que la historia del desarrollo del libreto de esta obra nos muestra un texto aún en progreso.

El libro de Christopher Stray, demuestra completamente el conocimiento enciclopédico del autor acerca de lo que ha sucedido "detrás de escena" para llegar al estado actual de los estudios clásicos en Gran Bretaña. Se trata de un ambicioso recorrido, que no deja arista sin atender y que permite reconstruir este trayecto con lujo de detalles. Sin duda, una lectura recomendable para estudiantes y especialistas interesados por ahondar en las raíces de los estudios clásicos británicos.

\section{Leandro Nahuel Fernández Roveda \\ Universidad Nacional de Córdoba}

Infr1707@hotmail.com

Para citar esta reseña:

Fernández Roveda, Leandro Nahuel (2020): Reseña bibliográfica de: “Stray, C. (2018). Classics in Britain: Scholarship, Education, and Publishing 1800-2000. Oxford: Oxford University Press. xxvi, 385 pp." Anuario de la Escuela de Historia Virtual 17, 145-149. 